\title{
A Call to Arms: Surgeons Must Play an Important and Early Role in the Management of Patients with Advanced Melanoma
}

\author{
Michael C. Lowe, MD (D), and Keith A. Delman, MD \\ Department of Surgery, Emory University School of Medicine, Atlanta, GA
}

As systemic therapies continue to decrease recurrences and improve survival in patients with advanced melanoma, the management of patients with metastatic disease has evolved into a much more multidisciplinary approach. While a significant focus of recent literature has been on the success of novel systemic agents, incorporating surgery into the care of the patient with advanced melanoma is both increasing in frequency and dramatically impacting survival. Two articles published in this issue of Annals of Surgical Oncology demonstrate the role of surgery as an important component of the multidisciplinary approach to patients with both regional and distant metastatic disease. $^{1,2}$ In the analysis by Nelson et al. ${ }^{1}$ the 5 -year melanoma-specific survival (MSS) of stage IV patients who underwent surgery and received either immunotherapy or BRAF-targeted therapies was $45.5 \%$. In a matched-pair analysis of patients undergoing upfront surgery compared with patients undergoing modern systemic therapy alone, 5-year MSS was even better for the upfront metastasectomy group (58.8\% compared with $38.9 \%$ in the systemic therapy-only group). Furthermore, the study reported from the University of Pennsylvania group demonstrated that survival for patients with clinical stage III disease is markedly improved since the approval of immunotherapy. ${ }^{2}$ On cursory review, the juxtaposition of these two studies would imply that the credit should be applied to systemic therapy; however, it is important to consider that effective

(C) Society of Surgical Oncology 2019

First Received: 2 July 2019;

Published Online: 29 August 2019

M. C. Lowe, MD

e-mail: mlowe3@emory.edu systemic therapy often permits the surgeon to consider a more aggressive approach. The introduction of neoadjuvant therapeutic paradigms makes this increasingly true.

While the patients selected for surgery in the analysis by Nelson et al. ${ }^{1}$ were highly selected, $71.2 \%$ of patients undergoing metastasectomy did so before initiating systemic therapy. The matched-pair analysis mitigated some of the selection bias that might determine the surgeon's decision to perform metastasectomy. However, only disease-free interval and number of organs involved were matched between the upfront surgery and no-surgery groups. This approach allowed for important factors, such as age, comorbidities, and M stage (M1a vs. M1d) not to be taken into consideration when calculating differences in survival between these groups. Importantly, upfront surgery patients were on average almost 12 years younger than patients undergoing systemic therapy alone. Despite this limitation, these data demonstrate that surgical resection is an impactful intervention even in the setting of effective systemic therapy, particularly when used as firstline therapy.

This is not the first study to demonstrate an increase in the frequency of metastasectomy for stage IV patients. It does however highlight an important trend: The criteria used to select patients for surgery have loosened considerably since the introduction of effective systemic agents. In the historical cohort, female sex, no history of stage III disease, single-organ involvement, and M1a versus M1c disease were all independently associated with selection to undergo surgery. In the modern cohort, only age younger than 60 was independently related to the decision to perform surgery. This suggests that metastasectomy is being offered to more patients regardless of clinicopathologic factors that historically would have precluded patients from 
undergoing surgery. Despite these less stringent selection criteria, melanoma-specific survival continues to improve for patients undergoing surgery.

The decision to perform metastasectomy in the setting of stage IV disease needs to be discussed thoroughly amongst the medical and surgical teams. It is not novel to consider surgery in the treatment of patients with metastatic disease, but as data emerge that suggest that lower burden of disease is associated with higher efficacy of systemic therapies, the surgeon must be a vocal advocate for surgical eradication of known disease before the initiation of systemic therapy. ${ }^{3,4}$ Additionally, the surgeon may be the ideal individual to champion studies in this arena. Of course, surgery will not alter the course of disease in all patients, particularly older patients with a heavier burden of disease, but the combination of surgery and modern systemic therapies has proven to be a promising approach.

While the manuscript by Song et $\mathrm{al}^{2}$ in which survival was analyzed for clinical stage III patients undergoing surgery followed by adjuvant systemic therapy, focuses on a different population than the Nelson manuscript, the improvements in survival offer additional support for aggressive, multidisciplinary interventions in patients with advanced melanoma. On a population level (using the National Cancer Database), overall survival significantly increased in a modern cohort of patients (median 58.2 months) compared with a historic cohort for whom modern immune and targeted therapies were not available (median 49.3 months). While there are inherent limitations to analyses of large databases, such as NCDB, this study reinforces the notion that surgery followed by modern systemic therapy is changing the trajectory of management of patients with advanced melanoma.

With the advent of effective systemic therapy, and the recent publication of the DeCOG and MSLT-II studies, there has been considerable discussion about the decreasing role of surgery in the care of the melanoma patient. ${ }^{5,6}$ The two studies published in this issue of Annals of Surgical Oncology, along with the emerging data on the role of neoadjuvant approaches, salvage surgery and combination approaches, argue for a different approach. ${ }^{1,2}$ While the nature of surgical intervention may continue to evolve, surgery continues to play an increasingly important role in the multidisciplinary management of patients with complicated advanced melanoma.
Unfortunately, most of the data published to date on metastasectomy has been retrospective or in the context of clinical trials performed without modern systemic therapies, as is true with the studies highlighted here. Armed with the data published to date, surgeons need to increase awareness of the importance of surgical intervention at the time of diagnosis of stage III and IV disease. Surgeons must play a larger part in the design of prospective clinical trials that evaluate the benefit of combining surgery with systemic therapy. This includes determining which patients should be included based on extent of disease, ease of resectability, aggressiveness of tumor biology, and clinical factors. Similar to how the inclusion criteria of the only prospective adjuvant radiation trial to date has defined which patients should get adjuvant radiation, surgeons need to design clinical trials with inclusion criteria that more explicitly define which patients should get upfront surgery for stage IV melanoma. Then, and only then, will we be able to make the claim that surgery may be as important for stage IV melanoma patients as any modern systemic therapy approved to date.

\section{REFERENCES}

1. Nelson DW, Fischer TD, Graff-Baker AN, et al. Impact of effective systemic therapy on metastasectomy in stage iv melanoma: a matched-pair analysis. Ann Surg Oncol. 2019. http s://doi.org/10.1245/s10434-019-07487-5.

2. Song Y, Tieniber AD, Gimotty PA, et al. Survival outcomes of patients with clinical stage III melanoma in the era of novel systemic therapies. Ann Surg Oncol. 2019. https://doi.org/10.1245/ s10434-019-07599-y.

3. Hsueh EC, Essner R, Foshag LJ, Ollila DW, Gammon G, O’Day SJ, et al. Prolonged survival after complete resection of disseminated melanoma and active immunotherapy with a therapeutic cancer vaccine. J Clin Oncol. 2002;20(23):4549-54.

4. Ollila DW, Essner R, Wanek LA, Morton DL. Surgical resection for melanoma metastatic to the gastrointestinal tract. Arch Surg. 1996;131(9):975-9; 9-80.

5. Leiter U, Stadler R, Mauch C, Hohenberger W, Brockmeyer N, Berking $\mathrm{C}$, et al. Complete lymph node dissection versus no dissection in patients with sentinel lymph node biopsy positive melanoma (DeCOG-SLT): a multicentre, randomised, phase 3 trial. Lancet Oncol. 2016;17(6):757-67.

6. Faries MB, Thompson JF, Cochran AJ, Andtbacka RH, Mozzillo $\mathrm{N}$, Zager JS, et al. Completion dissection or observation for sentinel-node metastasis in melanoma. $N$ Engl $J$ Med. 2017;376(23):2211-22.

Publisher's Note Springer Nature remains neutral with regard to jurisdictional claims in published maps and institutional affiliations. 